\title{
Endothelin inhibitors lower pulmonary vascular resistance and improve functional capacity in patients with Fontan circulation
}

Gabriella Agnoletti, PhD, ${ }^{\text {a }}$ Simona Gala, MD, ${ }^{\mathrm{a}}$ Francesca Ferroni, MD, ${ }^{\mathrm{a}}$ Roberto Bordese, MD, Lorenzo Appendini, $\mathrm{MD},{ }^{\mathrm{b}}$ Carlo Pace Napoleone, $\mathrm{MD},{ }^{\mathrm{c}}$ and Laura Bergamasco, $\mathrm{PhD}^{\mathrm{b}}$

\section{ABSTRACT}

Objectives: To evaluate the effects of endothelin inhibitors (ERAs) on hemodynamic and functional parameters in patients post-Fontan procedure with high pulmonary vascular resistance (PVR).

Methods: Among our cohort of patients with Fontan circulation, 8 children, 8 adolescents, and 8 adults had PVR $\geq 2 \mathrm{WU} * \mathrm{~m}^{2}$. These patients were treated with ERAs (minors with bosentan, adults with macitentan) and reevaluated after 6 months. Pre- and posttreatment hemodynamic variables were assessed by cardiac catheterization. Functional capacity was evaluated by cardiopulmonary exercise testing (CPET). Our primary endpoint was to obtain a reduction of PVR; the secondary endpoint was to obtain an improvement of functional capacity.

Results: Under treatment, New York Heart Association class improved for adolescents and adults. PVR decreased $(P=.01)$ in all groups: in children from the median value 2.3 (interquartile range 2.0-3.1) to $1.9(1.4-2.3) \mathrm{WU}^{*} \mathrm{~m}^{2}$, in adolescents from $2.3(2.1-2.4)$ to $1.7(1.4-1.8) \mathrm{WU}^{*} \mathrm{~m}^{2}$, and in adults from 2.8 $(2.0-4.7)$ to $2.1(1.8-2.8) \mathrm{WU}^{*} \mathrm{~m}^{2}$. In $71 \%$ of patients, PVR fell to less than 2 WU* $\mathrm{m}^{2}$. Cardiac index increased in adolescents from $2.6(2.4-3.3)$ to 3.6 (3.4-4.3) $\mathrm{L} / \mathrm{min} / \mathrm{m}^{2}, P=.04$, and in adults from $2.1(2.0-2.3)$ to $2.8(2.3-4.7)$ $\mathrm{L} / \mathrm{min} / \mathrm{m}^{2}, P=.03$. CPET showed that only adolescents displayed a significant functional improvement. Anaerobic threshold improved from 17 (13-19) to 18 (13-20) $\mathrm{mL} / \mathrm{kg} / \mathrm{min}, P=.03$; oxygen consumption and $\mathrm{VO}_{2}$ max increased from $1.3(1.0-1.6)$ to $1.7(1.1-1.9) \mathrm{L} / \mathrm{min}, P=.02$ and from 25 (21-28) to 28 (26-31) L/min, $P=.02$, respectively. Oxygen pulse increased from 7.9 (5.7$10.4)$ to $11.2(8.2-13.0) \mathrm{L} /$ beat, $P=.01$.

Conclusions: This is the first study that assesses by cardiac catheterization and CPET the effects of ERA in patients with Fontan circulation with increased PVR. These results suggest that ERAs might provide most pronounced hemodynamic and functional improvement in adults and adolescents. (J Thorac Cardiovasc Surg 2017;153:1468-75)

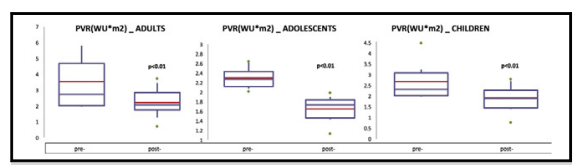

Endothelin inhibitors significantly decreased pulmonary vascular resistance in all 3 groups of patients.

\section{Central Message}

Our results suggest that endothelin inhibitors might be useful in patients with Fontan circulation and increased pulmonary vascular resistance. They provide the most pronounced hemodynamic and functional improvement in adults and adolescents (Video 1).

\section{Perspective}

We show that endothelin inhibitors normalize pulmonary vascular resistance in the majority of patients with Fontan circulation, increasing cardiac output. They improve ventilatory capacity and oxygen consumption. They induce most pronounced hemodynamic and functional improvement in adults and adolescents. We provide evidence that pulmonary vasodilatation with endothelin inhibitors might be useful in patients with Fontan circulation and increased pulmonary vascular resistance.

See Editorial Commentary page 1476.

See Editorial page 1466.
In patients with Fontan circulation, pulmonary vascular resistance (PVR) increases and indexed systemic output (QSI) slowly decreases with time since operation, eventually leading to Fontan failure and death. ${ }^{1,2}$ It is known

From the Divisions of a Pediatric Cardiology, ${ }^{\mathrm{b}}$ Statistics, and ${ }^{\mathrm{c}}$ Cardiac Surgery, Citta' della Salute e della Scienza, Turin, ASL CN1, Saluzzo, Italy.

Received for publication Aug 29, 2016; revisions received Jan 4, 2017; accepted for publication Jan 24, 2017; available ahead of print March 7, 2017.

Address for reprints: Gabriella Agnoletti, PhD, Servizio di Cardiologia Pediatrica, piazza Polonia, 10124 Torino, Italy (E-mail: gagnoletti@cittadellasalute.to.it). $0022-5223 / \$ 36.00$

Copyright (c) 2017 by The American Association for Thoracic Surgery

http://dx.doi.org/10.1016/j.jtcvs.2017.01.051 that patients with Fontan circulation display endothelial dysfunction, ${ }^{3}$ characterized by decreased production of nitric oxide ${ }^{4}$ and increased levels of endothelin. ${ }^{5}$ Various authors have hypothesized that drugs that lower PVR through an endothelial action, such as endothelin receptor

Scanning this QR code will take you to a supplemental video for the article. 


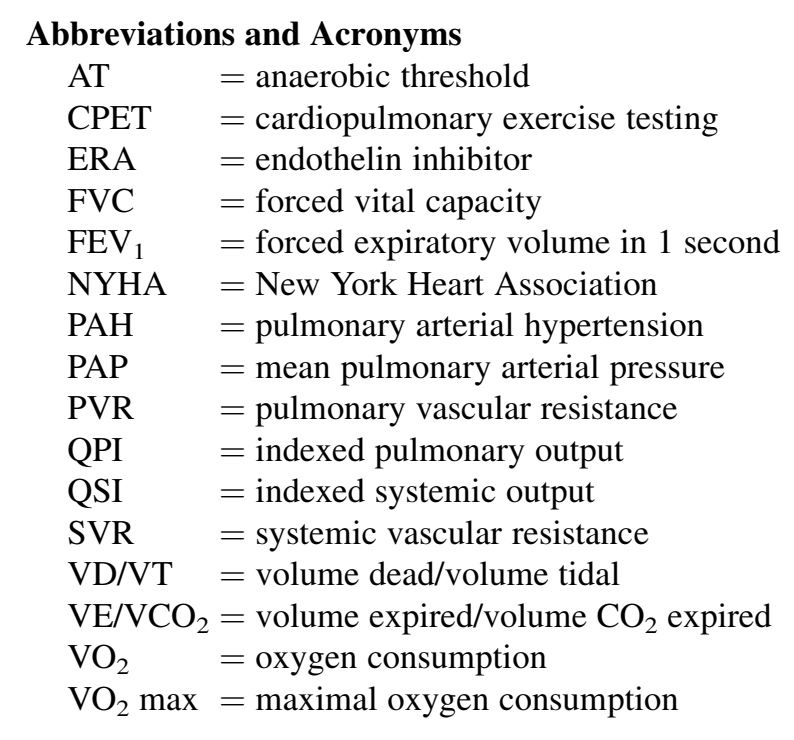

antagonists (ERAs) and phosphodiesterase-5 inhibitors, might improve symptoms and outcome of patients with Fontan circulation. ${ }^{6-9}$ The results concerning the effects of ERAs on the functional capacity of this population are, however, controversial. ${ }^{7-11}$ In particular, bosentan has been used in these patients with discordant results. Macitentan is a new ERA that was approved recently for the treatment of pulmonary arterial hypertension (PAH) in adult patients ${ }^{12}$ but so far has not been used in patients who had Fontan procedure. To the best of our knowledge, there are no studies exploring the effects of ERAs on PVR in patients with Fontan circulation.

The aim of the present study was to measure hemodynamic and functional parameters in a group of patients with Fontan circulation and increased PVR before and after treatment with ERAs for a period of 6 months.

\section{PATIENTS AND METHODS \\ Study Design}

The study was designed to explore the effect of a 6-month ERA treatment in patients who had Fontan procedure. Each patient was to act as case-control to test the impact of the ERA treatment. The primary end point was a reduction of PVR; the secondary end point was an improvement of functional capacity.

\section{Study Population}

The study population was derived from the pool of 64 consecutive patients who had Fontan procedure who were evaluated with cardiac catheterization and cardiopulmonary exercise testing (CPET) between March 2013 and December 2014 at our institution. ${ }^{1}$

Main inclusion criterion was PVR $\geq 2 \mathrm{WU}^{*} \mathrm{~m}^{2}$. Exclusion criteria were active plastic bronchitis, protein-losing enteropathy, or cirrhosis confirmed by liver biopsy. Patients in stable clinical condition, even with previous history of plastic bronchitis, protein-losing enteropathy, or with cirrhosis-like fibrosis, were included. No exclusion criteria were applied concerning either age or sex.

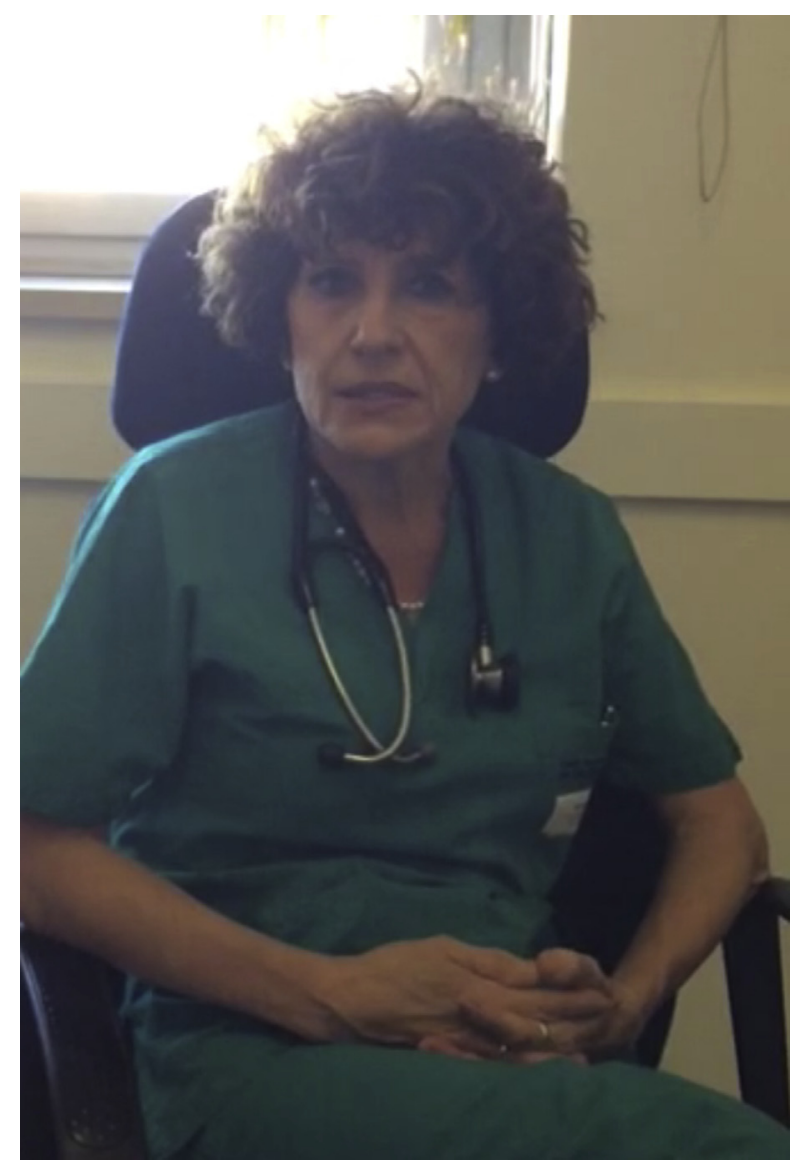

VIDEO 1. PVR increases over time in patients with Fontan circulation, leading to hypoperfusion and poor functional capacity. The aim of the present study was to measure hemodynamic and functional parameters in a group of patients with Fontan circulation with PVR $>2 \mathrm{Wu}^{*} \mathrm{~m}^{2}$ before and after treatment with ERAs for a period of 6 months. Pre- and posttreatment hemodynamic variables were assessed by cardiac catheterization. Functional capacity was evaluated by cardiopulmonary testing. Our primary endpoint was to obtain a reduction of PVR; our secondary endpoint was to obtain an improvement of functional capacity. We show for the first time that ERAs have positive functional and hemodynamic effects in patients with Fontan circulation of various ages with increased PVR. ERAs improve physical ability, ventilatory capacity, and oxygen consumption. They lower PVR, increasing cardiac index. Although functional capacity remains below average, PVR becomes normal in the majority of patients. Our overall results provide evidence that pulmonary vasodilatation with ERAs might be considered a useful treatment in patients with Fontan circulation with increased PVR. Video available at: http://www.jtcvsonline. org/article/S0022-5223(17)30201-5/addons.

Compliance to the study by adult patients or by parents of minors was strictly on a volunteer basis, following extensive information about its scope and procedures. Among the 26 of $64(41 \%)$ patients who met the PVR $\geq 2 \mathrm{WU}^{*} \mathrm{~m}^{2}$ condition, 24 of $26(92 \%)$ entered the ERA study: 16 minors (age $<18$ years) and 8 adults (age $\geq 18$ years).

\section{Study Protocol}

Although we aimed at administering macitentan to the entire population because of its improved receptorial binding, ${ }^{12}$ both the local legislative authority and the ethic committee allowed its use only in adult patients. Thus, 
minors received oral bosentan $0.5 \mathrm{mg} / \mathrm{kg}$ twice daily for 4 weeks, followed by $1 \mathrm{mg} / \mathrm{kg}$ twice daily for 5 months. Adults were treated with macitentan $10 \mathrm{mg}$ once daily.

Each patient had 1 visit at baseline, 1 visit for dose adjustment, and 1 visit at the end of the study. On each visit, adverse events (cutaneous reactions, cough, syncope, palpitations, vertigo), were recorded, and blood samples, including aminotransferases, international normalized ratio, hemoglobin, platelets, packed cell volume, urea, creatinine, and glomerular filtrate, were analyzed. On the baseline and on the dose-adjustment visit, acute tolerability was tested by measuring blood pressure, heart rate, and transcutaneous oxygen saturation before the first drug administration and again 1 and 2 hours afterwards. A decrease in systolic blood pressure $>20 \%$ or symptoms such as palpitations or vertigo were considered to reflect significant hypotension, thus leading to exclusion. Because none of the 24 patients reported side effects, none had to be excluded from the study.

Cardiac catheterization and CPET were performed before and after the treatment. The study started in March 2015 and was completed by May 2016. According to our institutional protocol, all patients (or parents of minors) were informed extensively about the clinical procedures and about the possible use of their data for study purposes and signed an informed consent form. Patients' information was anonymized before this analysis.

\section{Cardiac Catheterization}

The technique of catheterization did not differ from that described in the publication of Agnoletti and colleagues. ${ }^{13}$ General anesthesia was used in children and adolescents. Heparin at the dose of $100 \mathrm{UI} / \mathrm{kg}$ and antibiotic prophylaxis, when appropriate, were administered. Pressures, indexed pulmonary output (QPI), QSI, and PVR were obtained. When necessary, associated lesions were treated. All measurements were taken after treatment of associated lesions during the basal evaluation and before treatment of associated lesions during the evaluation performed under ERAs.

\section{Spirometry}

Immediately before each exercise test, patients underwent spirometric measurements of forced vital capacity (FVC), volume of air exhaled in $1 \mathrm{sec}-$ ond of forced expiration, or forced expiratory volume in 1 second $\left(\mathrm{FEV}_{1}\right)$, and the ratio volume dead/volume tidal (VD/VT). Heart rate, systolic and diastolic blood pressure, and oxygen saturation at rest also were measured.

\section{Cardiopulmonary Exercise Test}

Each patient used a bicycle ergometer (Ebike V2; GE, Fairfield, Conn). An appropriate incremental load protocol was chosen for each participant on the basis of expected fitness, with the purpose of reaching maximal effort within 6 to 12 minutes. All patients started with a 1- to 2-minute warm-up. All subjects were encouraged to exercise until exhaustion. A respiratory exchange ratio $>1.1$ was considered an indication that maximal effort was achieved.

Electrocardiographic monitoring and breath-by-breath expiratory gas analysis were performed with the $\mathrm{CardiO} 2$ exercise testing system (Erich Jaeger GmbH, Friedberg, Germany). Cuff blood pressure determinations and complete 12-lead electrocardiograms were obtained every 2- to 3minute intervals during exercise, at peak exercise, and 1,3, and 5 minutes after exercise. Pulse oximetry oxygen saturation also was monitored throughout the study. Oxygen consumption and minute ventilation were measured continuously. Outcome measures, calculated at maximal exercise, included workload, heart rate, systolic and diastolic blood pressure, oxygen saturation, peak minute oxygen consumption $\left(\mathrm{VO}_{2}\right)$, oxygen pulse (a surrogate for forward stroke volume) and arteriovenous oxygen difference at peak exercise, ${ }^{14}$ anaerobic threshold (AT), minute ventilation (VE) versus dioxide production ([VE/ $\left.\mathrm{VCO}_{2}\right]$, an index of the efficiency of gas exchange during exercise), VD/VT, and breathing reserve. AT was defined as the first 15 -second interval in which the respiratory exchange ratio exceeded 1 without subsequently falling below 1 . Maximal workload was the greatest watt load achieved for at least 15 seconds. Abnormal exercise capacity was defined as a level below $90 \%$ of predicted peak $\mathrm{VO}_{2}$.

\section{Statistical Analysis}

Continuous variables were checked for normality with the KolmogorovSmirnov-Lilliefors test and the Shapiro-Wilk W test. Normality was rejected for a large part of variables, so, for simplicity we chose to express all of them as median and interquartile range (25th percentile- 75 th percentile) and to use nonparametric tests for their comparison. Matched data (pre-post) were compared with the Wilcoxon test; independent data were compared with the Mann-Whitney $U$ test. Statistical significance was set at 2-tails $P<.05$. Open source software (www.openepi.com and www. vassarstats.net) and Statplus, Mac version v6 (AnalystSoft, Walnut, Calif) were used.

\section{RESULTS \\ Clinical Data}

The baseline features of patients are shown in Table 1, subdivided in 3 age groups: children (age $<15$ years), adolescents ( $15 \leq$ age $\leq 18$ years), and adults (age $>18$ years). The majority of adults and adolescents had left-type ventricles, whereas in children left-type and right-type ventricles were represented similarly. Adult patients underwent Fontan completion at an older age compared with adolescents $(P=.09)$ and children $(P=.02)$. Oxygen saturation was $\leq 90 \%$ in 5 patients. Five patients, stable during the study period, had a history of plastic bronchitis $(\mathrm{n}=3)$ or

TABLE 1. Clinical characteristics of patients before treatment

\begin{tabular}{lccc}
\hline & Children (bosentan) & Adolescents (bosentan) & Adults (macitentan) \\
\hline $\mathrm{n}$ & 8 & 8 & 8 \\
Female patients & $4 / 8$ & $1 / 8$ & $3 / 8$ \\
Age, y & $9.0(6.2 ; 12.0)$ & $16.5(15.0 ; 17.0)$ & $25.5(18.0 ; 31.7)$ \\
Weight, kg & $27.5(19.6 ; 33.5)$ & $43.0(38.0 ; 59.7)$ & $64.0(51 ; 77)$ \\
BSA, m ${ }^{2}$ & $1.02(0.80 ; 1.18)$ & $1.39(1.31 ; 1.71)$ & $1.75(1.50 ; 1.89)$ \\
Type of ventricle (L, R) & $3 \mathrm{~L}, 3 \mathrm{R}, 2 \mathrm{NA}$ & $6 \mathrm{~L}, 1 \mathrm{R}, 1 \mathrm{NA}$ & $5 \mathrm{~L}, 3 \mathrm{NA}$ \\
Age at TCPC, y & $3.5(3.2 ; 4.7)$ & $4.5(4.0 ; 5.7)$ & $8.0(4.7 ; 13.0)$ \\
Time interval since TCPC, y & $4.5(3.2 ; 7.5)$ & $11.5(10.2 ; 12.7)$ & $13.0(11.5 ; 16.5)$ \\
Oxygen saturation, \% & $96.5(94.5 ; 97.0)$ & $100(99.7 ; 100)$ & $96(93.5 ; 97.0)$ \\
\hline Conting
\end{tabular}

Continuous variables are reported as median (25th percentile-75th percentile). $B S A$, Body surface area; $L$, left-type; $R$, right-type; $N A$, not attributable; $T C P C$, total cavopulmonary connection. 
protein-losing enteropathy $(n=2)$. Five patients had cirrhosis-like fibrosis with preserved synthesis. In 3 patients, the fenestration was open.

Seventy-five percent of adolescents were in New York Heart Association (NYHA) class I, $12.5 \%$ in NYHA class II, and $12.5 \%$ in NYHA class III; for adults the percentages were $37.5 \%, 37.5 \%$, and $25 \%$, respectively (Figure 1: data labeled as "pre"). Figure 1 shows that the ERA treatment (data labeled as "post") upgraded both adolescents and adults to a higher class of the NYHA class distribution, eliminating NYHA class III.

Systolic blood pressure diminished in all 3 groups, significantly so only for adolescents $(P=.03)$. No significant differences were detected in diastolic blood pressure and oxygen saturation (Table 2). Blood tests of hepatic and renal function were unchanged, no hepatic toxicity developed, and anemia never occurred.

\section{Hemodynamic Data}

Table 3 shows the relevant hemodynamic data pre- and posttreatment. At baseline adults had significantly greater systemic vascular resistance (SVR) than children $(P=.04)$ and adolescents $(P=.003)$, significantly lower than QPI children $(P=.03)$ and adolescents $(0.02)$, whereas QSI was statistically greater for adolescents $(P=.04)$ but not for children $(P=.16)$. The mean pulmonary arterial pressure (PAP), PVR, and wedge pressure were similar $(.30<P \leq .50, .50 \leq P \leq .90$ and $.40<P<.90$, respectively).

Figure 2 shows that ERAs significantly reduced PVR in all 3 age groups $(P=.01)$ with similar variations (about $-30 \%$ ) from the pre- to posttreatment. In $71 \%$ of patients (4/8 children, 8/8 adolescents, 4/8 adults), PVR decreased to less than $2 \mathrm{WU}^{*} \mathrm{~m}^{2}$.

\section{NYHA class}

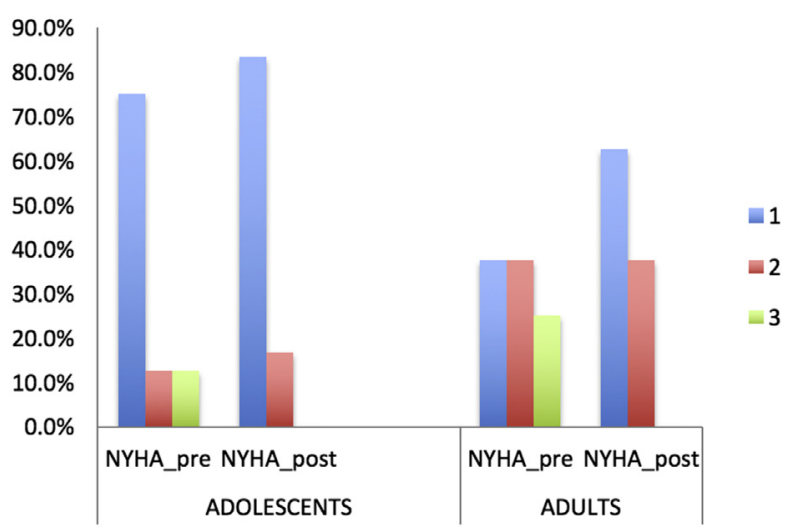

FIGURE 1. After treatment with endothelin inhibitors (data labeled as "post") the NYHA class distribution improved for both adolescents and adults, with all patients shifted to a greater class. NYHA, New York Heart Association.
Table 3 reports for each group other relevant pre- and posttreatment values. Under treatment, both QSI and QPI increased; statistical significance was reached for the former in children $(P=.03)$ and adults $(P=.002)$ and for the latter in adolescents $(P=.04)$ and adults $(P=.03)$. The mean PAP, wedge pressure, ratio $\mathrm{QP} / \mathrm{QS}$, and SVR were unchanged for all groups.

Sixteen interventions were performed in 15 patients: embolization of aortopulmonary collateral vessels in 3 , embolization of venous vessels prompting cyanosis in 8 , and stenting of pulmonary arteries in 5 .

\section{Spirometric Data}

The pre- and posttreatment data are shown in Tables 2 and 4 . Three patients ( 2 children and 1 adult) could not complete the study because of physical inability: spirometric data were thus available for 6 of 8 children, 8 of 8 adolescents, and 7 of 8 adults.

In basal conditions, FVC and $\mathrm{FEV}_{1}$ were less than $90 \%$ of the predicted value in $90 \%$ and $62 \%$ of patients, respectively. FVC and $\mathrm{FEV}_{1}$ generally improved after the ERA treatment, with a statistically significant increase for children $(P=.03)$ and adolescents $(P=.01)$. The VD/VT ratio was within normal limits in all patients and, under treatment, significantly increased for adolescents $(P=.03)$. Systolic blood pressure significantly diminished in adolescents, whereas diastolic blood pressure was unchanged in all groups. Basal oxygen saturation did not change under treatment.

\section{CPET Data}

The results obtained at peak exercise are shown in Table 4. Nine patients interrupted the test due to fatigue, 9 due to dyspnea, and 3 due to desaturation. Three patients had ectopic ventricular beats. Five patients performed a maximal test, 8 displayed mild-to-moderate, and 8 a severe reduction of their functional capacity. Under treatment, the maximal workload improved in all 3 groups, reaching borderline statistical significance for children $(P=.046)$. Maximal heart rate, systolic and diastolic blood pressure, and oxygen saturation were unchanged. Adolescents, but not children, showed a significant increase of AT $(P=.03), \mathrm{VE} / \mathrm{VCO}_{2}(P=.02)$, and oxygen pulse $(P=.01)$. In the adult group, all values increased mildly but well under significance. Before treatment, the AT was identified in 14 of 21 patients and, after treatment, in all. The breathing reserve in basal condition was $<20 \%$ in 2 of $21(9.5 \%)$ of patients (one child and one adult): both of them went greater than $20 \%$ at peak exercise, when the number of patients with breathing reserve less than $20 \%$ amounted to 4 of $21(19 \%$ ) (2 children and 2 adolescents). $\mathrm{VO}_{2}$ significantly increased in children and adolescents, whereas maximal oxygen consumption 
TABLE 2. Spirometric data at rest before and after treatment with endothelin inhibitors

\begin{tabular}{|c|c|c|c|c|c|c|c|c|c|}
\hline \multirow[b]{2}{*}{ Value } & \multicolumn{3}{|c|}{ Children (bosentan), $n=6$} & \multicolumn{3}{|c|}{ Adolescents (bosentan), $\mathbf{n}=\mathbf{8}$} & \multicolumn{3}{|c|}{ Adults (macitentan), $n=7$} \\
\hline & Pre & Post & $\boldsymbol{P}$ & Pre & Post & $P$ & Pre & Post & $P$ \\
\hline $\mathrm{FVC}, \mathrm{L}$ & $1.8(1.4-2.1)$ & $2.1(1.8-2.4)$ & $.03 *$ & $3.1(1.6-3.7)$ & $3.4(3.0-4.0)$ & $.01 *$ & $2.4(2.0-4.2)$ & $3.0(2.0-4.0)$ & .18 \\
\hline$\%$ predicted value & $77(73-82)$ & $88(85-97)$ & $.03 *$ & $75(57-80)$ & $90(72-106)$ & $.01 *$ & $82(50-91)$ & $67(56-78)$ & .18 \\
\hline $\mathrm{FEV}_{1}, \mathrm{~L} / 1 \mathrm{~min}$ & $1.7(1.3-2.0)$ & $1.8(1.4-2.2)$ & $.03 *$ & $2.7(1.6-3.5)$ & $3.0(2.1-3.7)$ & $.01 *$ & $2.4(1.9-3.7)$ & $2.5(1.7-3.7)$ & .74 \\
\hline$\%$ predicted value & $84(75-93)$ & $91(82-101)$ & $.03 *$ & $75(64-90)$ & $89(80-103)$ & $.01 *$ & $89(59-93)$ & $80(55-97)$ & .74 \\
\hline $\mathrm{VD} / \mathrm{VT}$ & $0.35(0.29-0.40)$ & $0.34(0.27-0.43)$ & .92 & $0.34(0.29-0.40)$ & $0.40(0.30-0.50)$ & $.02 *$ & $0.33(0.20-0.41)$ & $0.39(0.29-0.46)$ & .06 \\
\hline HR, bpm & $71(67-87)$ & $75(62-88)$ & .79 & $70(64-88)$ & $76(54-87)$ & .80 & $76(68-79)$ & $73(68-87)$ & .93 \\
\hline $\mathrm{SBP}, \mathrm{mm} \mathrm{Hg}$ & $102(93-114)$ & $99(90-111)$ & .46 & $115(110-128)$ & $91(80-100)$ & $.03 *$ & $120(110-125)$ & $115(105-120)$ & .35 \\
\hline $\mathrm{DBP}, \mathrm{mm} \mathrm{Hg}$ & $70(66-73)$ & $77(65-82)$ & .22 & $77(65-95)$ & $77(60-80)$ & .53 & $80(70-80)$ & $70(70-80)$ & .08 \\
\hline $\mathrm{O}_{2}$ saturation, $\%$ & $97(94-98)$ & 95 (94-97) & .14 & $93(89-97)$ & $92(89-97)$ & .93 & 95 (94-96) & $93(92-97)$ & .18 \\
\hline
\end{tabular}

Data are reported as median (25th percentile-75th percentile). $F V C$, Forced vital capacity; $F E V_{l}$, forced expiratory in 1 second; $V D / V T$, volume dead/volume tidal; $H R$, heart rate; $S B P$, systolic blood pressure; $D B P$, diastolic blood pressure. *Statistical significance.

$\left(\mathrm{VO}_{2} \mathrm{max}\right)$ significantly increased only in adolescents. Oxygen pulse significantly increased only in adolescents. The adolescent group seemed to be the one that most profited from the treatment, with 6 significant variations (AT, VE/ $\mathrm{VCO}_{2}$, breathing reserve, $\mathrm{VO}_{2}, \mathrm{VO}_{2}$ max, and oxygen pulse); the children group had $1\left(\mathrm{VO}_{2}\right)$ and the adult group had none.

\section{DISCUSSION}

This is the first study to show positive effects of both bosentan and macitentan on clinical, hemodynamic, and functional conditions of patients with single-ventricle and elevated PVR. In the Fontan circulation, pulmonary flow is driven without support by a subpulmonary ventricle. Thus, even a slight increase in PVR may compromise the pulmonary circulation, lower the preload of the ventricle, and limit the cardiac output and exercise tolerance. ${ }^{15}$ In addition, an increase in PVR worsens liver fibrosis, typically associated with Fontan circulation, ${ }^{16}$ and is poorly tolerated by hearts with an increased myocardial fibrogenesis and impaired ventricular diastolic function. ${ }^{17}$

It is known that PVR increase over time after Fontan completion $^{1}$ and that increased levels of endothelin-1 correlate with increased PVR and failure of the Fontan circulation. ${ }^{4}$ Thus, pulmonary vasodilators that predominantly modulate endothelial function could theoretically improve exercise-dependent hemodynamic requirements, exercise tolerance, and well being in these patients.

Unlike patients with $\mathrm{PAH},{ }^{18}$ patients with Fontan circulation have mildly elevated PVR. In these patients, several factors may contribute to exercise limitations and account for treatment effects lower than for PAH.

Several authors used phosphodiesterase- 5 inhibitors, ERAs, or prostanoids to decrease PVR in patients with Fontan circulation and improve their functional capacity. ${ }^{6-9}$ Studies demonstrating that these drugs actually lower PVR and PAP in patients with Fontan circulation are, however, scanty. In a retrospective study, ${ }^{19}$ bosentan $(\mathrm{n}=14)$ or sildenafil $(\mathrm{n}=20)$ was administered to patients with univentricular physiology before $(\mathrm{n}=18)$ or after $(n=16)$ Fontan. Measurements of PVR and PAP after treatment were, however, available only for 6 of 14 and 11 of 20 patients, respectively. In addition, the study did not specify which patients were treated with bosentan and, if so, whether before or after Fontan completion. In a prospective, multicenter study, sildenafil significantly decreased PVR in 42 patients treated before or after Fontan. ${ }^{6}$ In an open-label study of 27 patients randomized to either a single oral dose of sildenafil $(n=18)$ or no treatment $(\mathrm{n}=9)$, sildenafil increased pulmonary blood flow and peak $\mathrm{VO}_{2} \cdot{ }^{20}$ These findings, however, could not be

TABLE 3. Hemodynamic data: pre- and posttreatment values

\begin{tabular}{|c|c|c|c|c|c|c|c|c|c|}
\hline \multirow[b]{2}{*}{ Value } & \multicolumn{3}{|c|}{ Children (bosentan), $\mathbf{n}=8$} & \multicolumn{3}{|c|}{ Adolescents (bosentan), $\mathbf{n}=\mathbf{8}$} & \multicolumn{3}{|c|}{ Adults (macitentan), $n=8$} \\
\hline & Pre & Post & $\boldsymbol{P}$ & Pre & Post & $\boldsymbol{P}$ & Pre & Post & $\boldsymbol{P}$ \\
\hline $\mathrm{PVR}, \mathrm{WU} * \mathrm{~m}^{2}$ & $2.3(2.0-3.1)$ & $1.9(1.4-2.3)$ & .01 & $2.3(2.1-2.4)$ & $1.7(1.4-1.8)$ & .01 & $2.8(2.0-4.7)$ & $2.1(1.8-2.8)$ & .01 \\
\hline PAP, mm Hg & $12.0(11.7-13.5)$ & $12.0(10.7-14.5)$ & .83 & $12.0(10.7-13.0)$ & $10.0(8.7-13.0)$ & .31 & $15.5(10.7-17.5)$ & $11.5(8.0-15.0)$ & .06 \\
\hline Wedge pressure, $\mathrm{mm} \mathrm{Hg}$ & $6.0(5.0-7.2)$ & $6.0(5.7-8.0)$ & .86 & $6.0(4.7-7.2)$ & $5.0(4.0-6.2)$ & .75 & $7.5(5.0-10.5)$ & $5.5(5.0-8.0)$ & .33 \\
\hline $\mathrm{SVR}, \mathrm{WU} * \mathrm{~m}^{2}$ & $16.6(14.5-18.3)$ & $13.5(12.8-17.3)$ & .40 & $15.1(12.1-23.1)$ & $14.0(12.1-23.1)$ & .78 & $35.7(28.8-37.6)$ & $24.0(11.2-29.8)$ & .12 \\
\hline QPI, $\mathrm{L} / \mathrm{min} / \mathrm{m}^{2}$ & $2.5(2.3-2.8)$ & $3.4(2.9-3.9)$ & $.03 *$ & $2.5(2.3-2.8)$ & $3.3(2.8-3.5)$ & .07 & $1.9(1.6-2.4)$ & $2.6(1.8-3.7)$ & $.002 *$ \\
\hline QSI, $\mathrm{L} / \mathrm{min} / \mathrm{m}^{2}$ & $2.8(2.3-3.7)$ & $3.4(3.2-3.7)$ & .26 & $2.6(2.4-3.3)$ & $3.6(3.4-4.3)$ & $.04 *$ & $2.1(2.0-2.3)$ & $2.8(2.3-4.7)$ & $.03 *$ \\
\hline QPI/QSI & $0.89(0.83-0.99)$ & $0.94(0.86-1.00)$ & .09 & $0.95(0.92-1)$ & $0.85(0.82-0.95)$ & .21 & $0.94(0.83-1.03)$ & $0.95(0.68-1.07)$ & .57 \\
\hline
\end{tabular}

PVRs also are shown in Figure 2. Data are reported as median (25th percentile-75th percentile). $P V R$, Pulmonary vascular resistance; PAP, pulmonary arterial pressure; SVR, systemic vascular resistance; $Q P I$, pulmonary output; $Q S I$, systemic output. *Statistical significance. 


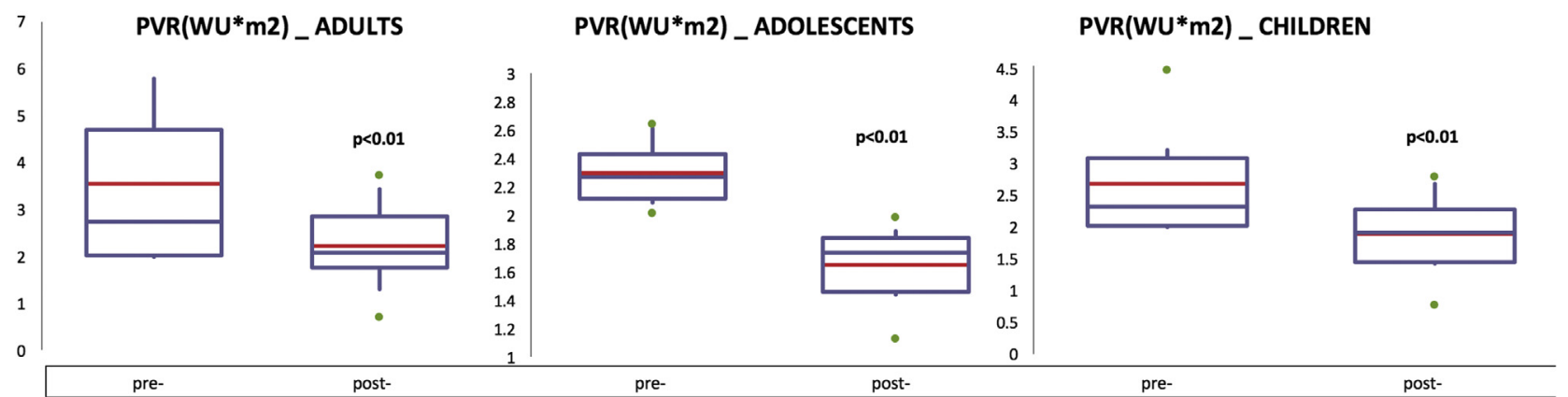

FIGURE 2. PVR significantly decreased in all 3 groups, with similar variations (about $-30 \%$ ) from the pre- to the posttreatment situation. $P V R$, Pulmonary vascular resistance.

confirmed in a subsequent randomized, double-blind, placebo-controlled trial with 28 patients. ${ }^{21}$

To the best of our knowledge, the effect of ERAs on PVR in patients with Fontan circulation has not yet been tested with a prospective study. Macitentan is a novel oral ERA, characterized by dual sustained receptor binding and enhanced tissue penetration. It was approved for the longterm treatment of patients with $\mathrm{PAH}$, to delay disease progression $^{22}$ and significantly reduce morbidity and mortality. ${ }^{23}$

Patients with Fontan circulation with increased PVR are fragile, and PVR tends to increase with time. Because of legislative reasons, however, we had to treat the younger cohort of patients with Bosentan.

\section{Hemodynamic Data}

Our cohort of patients was heterogeneous, and each age group comprised only 8 patients; we were thus surprised to find significant results concerning the pre- and posttreatment variables even within each group. Basal hemodynamic variables confirmed what we expected to find in patients of different age. Adults had significantly greater SVR and QSI than younger patients, whereas the remaining variables were similar.

The major and new finding of our study is that ERAs significantly decreased PVR after 6 months of treatment, with the majority of patients achieving the target of PVR $\leq 2 \mathrm{WU}^{*} \mathrm{~m}^{2}$. Although in adults, as expected, PVR were greater than in adolescents and children, the decrease of

TABLE 4. Peak cardiovascular responses before and after treatment with endothelin inhibitors

\begin{tabular}{|c|c|c|c|c|c|c|c|c|c|}
\hline \multirow[b]{2}{*}{ Values } & \multicolumn{3}{|c|}{ Children (bosentan), $n=6$} & \multicolumn{3}{|c|}{ Adolescents (bosentan), $n=8$} & \multicolumn{3}{|c|}{ Adults (macitentan), $n=7$} \\
\hline & Pre & Post & $\boldsymbol{P}$ & Pre & Post & $\boldsymbol{P}$ & Pre & Post & $\boldsymbol{P}$ \\
\hline Work load, W & $48(46-57)$ & $78(69-85)$ & .046 & $82(70-98)$ & $90(78-139)$ & .26 & $75(66-110)$ & $82(72-104)$ & .35 \\
\hline$\%$ predicted value & $48(39-70)$ & $70(58-102)$ & .046 & $44(33-60)$ & $55(34-75)$ & .16 & $43(36-64)$ & $50(39-52)$ & .87 \\
\hline HRmax, bpm & $141(105-160)$ & $156(153-168)$ & .12 & $163(146-174)$ & $151(135-168)$ & .44 & $146(134-159)$ & $135(106-148)$ & .47 \\
\hline$\%$ predicted value & $72(51-78)$ & $76(74-86)$ & .12 & $82(74-87)$ & $74(68-86)$ & .48 & $71(64-89)$ & $65(48-73)$ & .23 \\
\hline $\mathrm{SBP}, \mathrm{mm} \mathrm{Hg}$ & $140(134-143)$ & $135(120-145)$ & .47 & $137(125-145)$ & $136(133-155)$ & .78 & $136(130-150)$ & $135(125-160)$ & .45 \\
\hline $\mathrm{DBP}, \mathrm{mm} \mathrm{Hg}$ & $72(68-87)$ & $82(74-88)$ & .06 & $84(76-99)$ & $79(75-85)$ & .92 & $80(65-85)$ & $80(70-85)$ & .92 \\
\hline Oxygen saturation, $\%$ & $95(90-96)$ & $92(83-96)$ & .08 & $91(90-96)$ & $91(86-93)$ & .06 & $92(92-96)$ & $90(89-95)$ & .18 \\
\hline $\mathrm{AT}, \mathrm{mL} / \mathrm{kg} / \mathrm{min}$ & $21(17-28)$ & $18(16-22)$ & .11 & $17(13-19)$ & $18(13-20)$ & $.03 *$ & $14.5(8-19)$ & $15(8-19)$ & .74 \\
\hline$\%$ predicted value & $49(39-72)$ & $46(38-57)$ & .46 & $41(28-47)$ & $46(42-55)$ & $.03 *$ & $38(32-40)$ & $36(17-41)$ & .74 \\
\hline $\mathrm{VE} / \mathrm{VCO}_{2}, \%$ & $40(38-44)$ & $42(39-42)$ & .92 & $35(31-37)$ & $38(36-40)$ & $.03^{*}$ & $38(36-44)$ & $41(35-44)$ & .40 \\
\hline$\%$ predicted value & $165(157-172)$ & $163(150-178)$ & .92 & $146(138-168)$ & $160(151-188)$ & $.03 *$ & $193(167-233)$ & $187(163-235)$ & .40 \\
\hline $\mathrm{VD} / \mathrm{VT}, \%$ & $0.18(0.16-0.22)$ & $0.16(0.14-0.22)$ & .18 & $0.16(0.14-0.19)$ & $0.18(0.13-0.18)$ & .61 & $0.14(0.13-0.21)$ & $0.16(0.15-0.20)$ & .15 \\
\hline Breathing reserve, $\%$ & $45(27-57)$ & $37(15-46)$ & .08 & $47(30-60)$ & $58(11-68)$ & .40 & $49(40-55)$ & $52(41-59)$ & .60 \\
\hline $\mathrm{VO}_{2}, \mathrm{~L} / \mathrm{min}$ & $0.85(0.76-0.92)$ & $1.0(0.98-1.2)$ & $.03^{*}$ & $1.3(1.0-1.6)$ & $1.7(1.1-1.9)$ & $.02 *$ & $1.1(1.0-1.6)$ & $1.2(1.1-1.8)$ & .24 \\
\hline$\%$ predicted value & $66(48-90)$ & $67(58-84)$ & $.03 *$ & $56(48-67)$ & $66(52-83)$ & $.03 *$ & $55(48-62)$ & $54(51-62)$ & .24 \\
\hline $\mathrm{VO}_{2} \max$ & $30(19-36)$ & $28(26-35)$ & .92 & $25(21-28)$ & $28(26-31)$ & $.02 *$ & $23(19-29)$ & $24(19-26)$ & .34 \\
\hline$\%$ predicted value & $64(49-100)$ & $69(63-89)$ & .92 & $61(44-66)$ & $64(52-74)$ & $.02 *$ & $52(48-62)$ & $54(50-62)$ & .34 \\
\hline Oxygen pulse, $\mathrm{mL} /$ beat & $6.1(5.3-7.5)$ & $6.8(6.1-7.2)$ & .46 & $7.9(5.7-10.4)$ & $11.2(8.2-13.0)$ & $.01^{*}$ & $8.4(8.1-11.3)$ & $12.6(6.1-18.8)$ & .24 \\
\hline$\%$ predicted value & $85(58-128)$ & $80(68-128)$ & .75 & $85(58-128)$ & $80(68-128)$ & $.01 *$ & $62(55-73)$ & $78(57-121)$ & .24 \\
\hline
\end{tabular}

Data are reported as median (25th percentile-75th percentile). HRmax, Maximum heart rate; $S B P$, systolic blood pressure; $D B P$, diastolic blood pressure; $A T$, anaerobic threshold; $V E / V C O_{2}$, volume expired/volume $\mathrm{CO}_{2}$ expired; $V D / V T$, volume dead/volume tidal; $V O_{2}$, oxygen consumption, $V O_{2}$ max, maximal oxygen consumption. *Statistical significance. 
PVR was similar in the 3 groups. We think that it is not fortuitous that PVR fell below $2 \mathrm{WU}^{*} \mathrm{~m}^{2}$ in all adolescents whereas in only $50 \%$ of children and adults. Children with increased PVR have a severe disease, whereas adults have a disease of longer duration.

ERAs do not diminish PVR by lowering mean PAP or wedge pressure, but increasing QSI and/or QPI, without modifying the ratio QPI/QSI. Thus, we can speculate that these drugs can recruit nonperfused small vessels. This hypothesis might explain our findings concerning the results of spirometry and CPET. In the 3 groups of patients, ERAs have no effect on SVR, making their use safe, and possibly decelerating the vicious cycle, typical of Fontan circulation, in which PVR tend to increase and QSI to decrease over time.

\section{Functional Data}

Unlike the effects on PVR, there is ample evidence of the effects of ERAs on the functional capacity of patients with Fontan circulation. Numerous studies document that survivors of Fontan surgery almost invariably have diminished exercise capacity. ${ }^{7,14,24}$ The exercise function tends to decline during late adolescence, but it appears to stabilize during early adulthood. ${ }^{25}$ Often, patients suffer from poor exercise tolerance despite the presence of a relatively well-preserved ventricular function. ${ }^{24,25}$ Parameters of CPET were shown to be strongly related to increased risk of hospitalization. ${ }^{24}$ The results of CPET can be very different in patients of various ages and should be carefully interpreted. In 2015, a placebo-controlled study showed that treatment with bosentan did not improve exercise capacity in patients with Fontan circulation. ${ }^{7}$ Interestingly, 1 year later, a randomized study on 69 patients demonstrated the opposite, ie, that bosentan significantly improved exercise capacity, exercise time, and functional class, without serious adverse events or hepatotoxicity. ${ }^{10}$

Our study confirms that patients with Fontan circulation have a diminished exercise capacity. FVC and $\mathrm{FEV}_{1}$ were below normal limits in the majority of patients, supporting the belief that pulmonary dysfunction is common in patients after the Fontan procedure and is independently associated with reduced exercise capacity. ${ }^{26}$ Our results show that adolescents, more than children and adults, profit from the ERAs treatment, because of a combined effect on both ventilatory capacity and peripheral perfusion. Basal spirometric results demonstrate an improvement of ventilatory capacity (increased $\mathrm{FVC}$ and $\mathrm{FEV}_{1}$ ) in children and adolescents but not in adults.

Adolescents were the population more sensitive to the effects of ERAs, with significantly lower systolic blood pressure and greater VD/VT rate than before the treatment. We think that these results may have different explications. Adolescents are young people pushed to perform active physical activity and, in general, ashamed to perform worse than their schoolmates. In this group, the good results of spirometry can be explained by an improvement of the ventilation/perfusion mismatch, possibly due to the recruitment of not-perfused pulmonary segments. A simultaneous increase of VD/VT, completely reversed under exercise, might suggest that this mechanism is not totally effective after 6 months of treatment. Also in children functional results are positive but less evident under exercise. In adults, $\mathrm{FVC}$ and $\mathrm{FEV}_{1}$ were unchanged, possibly due either to physical deconditioning or advanced disease. In accordance with Hebert and colleagues, ${ }^{10}$ we confirmed that $\mathrm{VO}_{2}$ improved significantly in children and adolescents $\left(\mathrm{VO}_{2}\right.$ max only in adolescents), even though it still remained below normal values. An interesting finding is that only adolescents improved their $\mathrm{AT}, \mathrm{VE} / \mathrm{VCO}_{2}$ ratio, breathing reserve, and the oxygen pulse. ${ }^{14}$ The results obtained in adolescents confirm that this population performs better due to a decrease of PVR combined to both increased cardiac index and peripheral perfusion. Unlike previous studies, we did not find that a large subset had a pathologically low breathing reserve ${ }^{26}$; in addition, only after treatment were we able to identify the AT in all patients.

Taken together, these results show that ERAs do improve functional capacity in a class of severely untrained patients. A tailored exercise program could probably increase both maximal workload and minute ventilation in all groups of patients.

\section{CONCLUSIONS}

We show for the first time that ERAs have positive functional and hemodynamic effects in patients with Fontan circulation of various ages with increased PVR. ERAs improve physical ability, ventilatory capacity and oxygen consumption. They lower PVR, increasing QSI. Although functional capacity remains below average, PVR becomes normal in the majority of patients. Our overall results provide evidence that pulmonary vasodilatation with ERAs might be considered a useful treatment in patients with Fontan circulation with PVR $\geq 2 \mathrm{Wu}^{*} \mathrm{~m}^{2}$

\section{Limitations of the Study}

Our study has a few limitations. Because patients with Fontan circulation and increased PVR are rare, this is a small series and the fact that only 21 of 24 patients performed a CPET limits the robustness of our results. We cannot exclude that a study on a larger, randomized and more homogeneous population might provide different results. Our population was relatively young (two thirds of patients are minors). We had to administer bosentan in minors and macitentan in adults. Even if all measurements were taken after treatment of associated lesions during the basal evaluation and before treatment of associated lesions during the evaluation performed under ERAs, we 
cannot rule out the possible role of associated lesions on our results.

\section{Conflict of Interest Statement}

Authors have nothing to disclose with regard to commercial support.

We thank Salvatore and Gregorio Curello for their support and for writing assistance.

\section{References}

1. Agnoletti G, Ferraro G, Bordese R, Marini D, Gala S, Bergamasco L, et al. Fontan circulation causes early, severe liver damage. Should we offer patients a tailored strategy? Int J Cardiol. 2016;209:60-5.

2. Murtuza B, Hermuzi A, Crossland DS, Parry G, Lord S, Hudson M, et al. Impact of mode of failure and end-organ dysfunction on the survival of adult Fontan patients undergoing cardiac transplantation. Eur $J$ Cardiothorac Surg. 2017; $135-41$

3. Henaine R, Vergnat M, Bacha EA, Baudet B, Lambert V, Belli E, et al. Effects of lack of pulsatility on pulmonary endothelial function in the Fontan circulation. $J$ Thorac Cardiovasc Surg. 2013;146:522-9.

4. Khambadkone S, Li J, de Leval MR, Cullen S, Deanfield JE, Redington AN. Basal pulmonary vascular resistance and nitric oxide responsiveness late after Fontan-type operation. Circulation. 2003;107:3204-8.

5. Yamagishi M, Kurosawa H, Hashimoto K, Nomura K, Kitamura N. The role of plasma endothelin in the Fontan circulation. J Cardiovasc Surg (Torino). 2002; 43:793-7.

6. Mori H, Park IS, Yamagishi H, Nakamura M, Ishikawa S, Takigiku K, et al. Sildenafil reduces pulmonary vascular resistance in single ventricular physiology. Int J Cardiol. 2016;221:122-7.

7. Derk G, Houser L, Miner P, Williams R, Moriarty J, Finn P, et al. Efficacy of endothelin blockade in adults with Fontan physiology. Congenit Heart Dis. 2015; 10:E11-6.

8. Schuuring MJ, Vis JC, van Dijk AP, van Melle JP, Vliegen HW, Pieper PG, et al. Impact of bosentan on exercise capacity in adults after the Fontan procedure: a randomized controlled trial. Eur J Heart Fail. 2013;15:690-8.

9. Rhodes J, Ubeda-Tikkanen A, Clair M, Fernandes SM, Graham DA, Milliren CE, et al. Effect of inhaled iloprost on the exercise function of Fontan patients: a demonstration of concept. Int J Cardiol. 2013;168:2435-40.

10. Hebert A, Mikkelsen UR, Thilen U, Idorn L, Jensen AS, Nagy E, et al. Bosentan improves exercise capacity in adolescents and adults after Fontan operation. Circulation. 2014;130:2021-30.

11. Ovaert C, Thijs D, Dewolf D, Ottenkamp J, Dessy H, Moons P, et al. The effect of bosentan in patients with a failing Fontan circulation. Cardiol Young. 2009;19: $331-9$.
12. Gatfield J, Mueller Grandjean C, Sasse T, Clozel M, Nayler O. Slow receptor dissociation kinetics differentiate macitentan from other endothelin receptor antagonists in pulmonary arterial smooth muscle cells. PLoS One. 2012;7:e47662.

13. Agnoletti G, Bordese R, Corleto A, Gabbarini F, Marini D. Interventional cath eterization after total cavopulmonary connection: experience in 68 patients. J Interv Cardiol. 2012;25:622-7.

14. Oliveira RB, Myers J, Araújo CG. Long-term stability of the oxygen pulse curve during maximal exercise. Clinics (Sao Paulo). 2011;66:203-9.

15. La Gerche A, Gewillig M. What limits cardiac performance during exercise in normal subjects and in healthy Fontan patients? Int J Pediatr. 2010;2010:1-8.

16. Kutty SS, Zhang M, Danford DA, Hasan R, Duncan KF, Kugler JD, et al. Hepatic stiffness in the bidirectional cavopulmonary circulation: The Liver AdultPediatric-Congenital-Heart-Disease Dysfunction Study group. J Thorac Cardiovasc Surg. 2016;151:678-84.

17. Sugimoto M, Saiki H, Tamai A, Seki M, Inuzuka R, Masutani S, et al. Ventricular fibrogenesis activity assessed by serum levels of procollagen type III N-terminal amino peptide during the staged Fontan procedure. J Thorac Cardiovasc Surg. 2016;151:1518-26.

18. von Siebenthal C, Aubert JD, Mitsakis P, Yerly P, Prior JO, Nicod LP, et al. Hy pertension and indicators of right ventricular function. Front Med (Lausanne) 2016;3:23.

19. Park IS. Efficacy of pulmonary vasodilator therapy in patients with functionally single ventricle. Int Heart J. 2015;56:S26-30.

20. Giardini A, Balducci A, Specchia S, Gargiulo G, Bonvicini M, Picchio FM. Ef fect of sildenafil on haemodynamic response to exercise and exercise capacity in Fontan patients. Eur Heart J. 2008;29:1681-7.

21. Goldberg DJ, French B, McBride MG, Marino BS, Mirarchi N, Hanna BD, et al Impact of oral sildenafil on exercise performance in children and young adults after the Fontan operation: a randomized, double-blind, placebo-controlled, crossover trial. Circulation. 2011;123:1185-93.

22. Simonneau G, Channick RN, Delcroix M, Galiè N, Ghofrani HA, Jansa P, et al Incident and prevalent cohorts with pulmonary arterial hypertension: insight from SERAPHIN. Eur Respir J. 2015;46:1711-20.

23. Pulido T, Adzerikho I, Channick RN, Delcroix M, Galiè N, Ghofrani HA, et al SERAPHIN Investigators. Macitentan and morbidity and mortality in pulmonary arterial hypertension. N Engl J Med. 2013;369:809-18.

24. Diller GP, Giardini A, Dimopoulos K, Gargiulo G, Müller J, Derrick G, et al. Predictors of morbidity and mortality in contemporary Fontan patients: results from a multicenter study including cardiopulmonary exercise testing in 321 patients. Eur Heart J. 2010;31:3073-83.

25. Fernandes SM, McElhinney DB, Khairy P, Graham DA, Landzberg MJ, Rhodes J. Serial cardiopulmonary exercise testing in patients with previous Fontan surgery. Pediatr Cardiol. 2010;31:175-80.

26. Opotowsky AR, Landzberg ML, Earing MG, Wu FM, Triedman JK, Dawn AC, et al. Abnormal spirometry after the Fontan procedure is common and associated with impaired aerobic capacity. Am J Physiol Heart Circ Physiol. 2014;307:H110-7.

Key Words: congenital heart disease, pulmonary hypertension, hemodynamics, Fontan circulation 